\title{
Modular symmetry, the semicircle law, and quantum Hall bilayers
}

\author{
C. P. Burgess ${ }^{1,2, *}$ and B. P. Dolan ${ }^{3,4, \dagger}$ \\ ${ }^{1}$ Department of Physics and Astronomy, McMaster University, 1280 Main Street West, Hamilton, Ontario, Canada L8S $4 M 1$ \\ ${ }^{2}$ Perimeter Institute, 31 Caroline Street North, Waterloo, Ontario, Canada N2L 2 Y5 \\ ${ }^{3}$ Department of Mathematical Physics, National University of Ireland, Maynooth, Ireland \\ ${ }^{4}$ School of Theoretical Physics, Dublin Institute for Advanced Studies, 10 Burlington Road, Dublin 4, Ireland
}

(Received 24 January 2007; published 11 October 2007)

\begin{abstract}
There is considerable experimental evidence for the existence in quantum Hall systems of an approximate emergent discrete symmetry, $\Gamma_{0}(2) \subset \operatorname{SL}(2, Z)$. The evidence consists of the robustness of the tests of a suite of predictions concerning the transitions between the phases of the system as magnetic fields and temperatures are varied, which follow from the existence of the symmetry alone. These include the universality of and quantum numbers of the fixed points which occur in these transitions, selection rules governing which phases may be related by transitions, and the semicircular trajectories in the Ohmic-Hall conductivity plane which are followed during the transitions. We explore the implications of this symmetry for quantum Hall systems involving two charge-carrying fluids, and so obtain predictions both for bilayer systems and for single-layer systems for which the Landau levels have a spin degeneracy. We obtain similarly striking predictions which include the phases which are seen in these systems, as well as a prediction for semicircle trajectories which are traversed by specific combinations of the bilayer conductivities as magnetic fields are varied at low temperatures.
\end{abstract}

DOI: 10.1103/PhysRevB.76.155310

PACS number(s): 73.43.-f, 05.30.Fk, 02.20.-a

\section{INTRODUCTION}

Quantum Hall systems continue to provide remarkable laboratories for studying the rich dynamics which is possible for strongly correlated electron systems in two spatial dimensions. The now-classic studies of the many Hall plateaux found within systems for which only the first Landau level is occupied $^{1}$ were later supplemented by the discovery of new and remarkable phenomena in systems involving higher Landau levels, ${ }^{2}$ and the discovery of evidence for new superfluid phases in bilayer systems, involving two or more quantum Hall layers which are spaced sufficiently closely to allow interlayer coherence and correlations. ${ }^{3}$

Although the microscopic picture of the electron physics in these systems is well understood in terms of the Laughlin wave function ${ }^{4}$-at least for those systems for which only the first Landau level is occupied-it is also useful to have a purely long-wavelength description of the effective degrees of freedom which are relevant [in the renormalization-group (RG) sense] for the low-energy transport measurements. As is well known from the study of superconductors (for which the BCS theory can be understood as the relevant effective description for energies near the superconducting gap), ${ }^{5}$ such a long-distance description complements the microscopic picture by identifying the domain of validity of the lowenergy predictions and so thereby allowing a better understanding of the robustness of various low-energy phenomena.

For homogeneous quantum Hall systems the large number of available ground states (compared, for example, to a BCS superconductor) complicates the low-energy description of the theory, and the detailed form of the effective theory applicable at $\mathrm{mK}$ temperatures is not yet fully understood. It has been suggested by a number of authors ${ }^{6,7}$ that there is a large discrete group, $\Gamma_{0}(2) \subset \operatorname{SL}(2, Z)$, that maps between these ground states-using complex conductivities, $\sigma=\sigma_{x y}$ $+i \sigma_{x x}$, the map is

$$
\sigma \rightarrow \frac{a \sigma+b}{c \sigma+d},
$$

where $a, b, c$, and $d$ are integers constrained by $a d-b c=1$ and $c$ is even. This symmetry commutes with the scaling flow in a sense that will be explained below. Indeed, much of the data concerning the transitions between plateaux follows very robustly simply from the existence of this symmetry. ${ }^{8}$ (We call this a "symmetry" even though it relates distinct phases of the system, rather than commuting with the Hamiltonian describing low-energy fluctuations within any one phase. We use this name because the group is a symmetry of the scaling flow.) On the theoretical side this symmetry was first proposed on both phenomenological ${ }^{7}$ and then on more microscopic grounds, ${ }^{9}$ before being derived as a consequence of particle-vortex duality for the relevant charge carriers $^{10}$ and then on very general grounds for twodimensional conformal field theories. ${ }^{11}$ The increasing generality of the assumptions which enter into these derivations helps us to explain the surprisingly broad domain of validity of the observations, which apply well beyond the immediate vicinity of the critical points to which they were originally expected to be restricted. Indeed the more recent derivations show these symmetries even go beyond the domain of linear response, ${ }^{12}$ in agreement with observations. ${ }^{13}$

As yet, there has not been an exhaustive study of the implications of this emergent discrete symmetry for the physics of spin-degenerate Landau levels ${ }^{14}$ or of bilayer systems. It is the purpose of this paper to do so, and we find that the symmetry has a number of observational implications, including the following.

(1) Symmetry. In the single-layer case there are two classes of emergent symmetry, depending on whether or not the charge carriers are related by symmetry transformations to bosons or to fermions. ${ }^{10}$ The corresponding symmetry for the bilayer system is determined by which of these symme- 
tries applies to the individual layers in the limit where they are independent. Given that all known spin-split monolayer quantum Hall effect (QHE) systems appear to be described by the fermionic symmetry, $\Gamma_{0}(2) \subset \operatorname{SL}(2, Z)$, we concentrate for the most part in this paper on the generalization of the purely fermionic symmetry. By combining the symmetries of each separate layer with the symmetry of layer interchange we are led to a particular discrete subgroup $G \subset \mathrm{Sp}(4, Z)$. A similar analysis of the group which is appropriate to systems built from "bosonic" layers is straightforward, and is also presented in the event that such layers should be experimentally realized.

(2) Low-temperature fixed points. Since the symmetry commutes with the flow, it makes universal predictions for the kinds of fixed points which are possible at low temperatures. Unlike the monolayer case, there cannot be a one-toone relation between the experimental variablestemperature, $T$, magnetic field, $B$, interlayer separation, $d$, and charge carrier density, $n_{s}$ - and the six conductivities which are possible for composite systems built from bilayers (in principle there could be a one-to-one correspondence for identical bilayers, with only four independent conductivities, but we are not aware of any experimental data that systematically explore the full parameter range). Not all phases can be explored for a given bilayer sample as $T, B$, and $d$ are varied, and a consequence of this observation is that the possible attractive fixed points which are allowed by the symmetries can depend on other microscopic factors, such as the relative importance of tunneling and interlayer or intralayer Coulomb energies. We identify three classes of flow which are consistent with the bilayer symmetry, and identify the universal low-energy fixed points that each predicts. These include all of the known phases which have been observed for these systems, with one describing widely separated monolayers, and the other two corresponding to correlated bilayers in two situations distinguished by the relative size of the tunneling and interlayer Coulomb energies.

(3) Semicircles. In the single-layer case, when particlehole symmetry is present, knowledge of the temperature flow implied the system moved on semicircular trajectories within the conductivity plane when magnetic fields are varied at low temperatures. This conclusion has generalizations to the three categories of flows described above. In particular, for samples exhibiting particle-hole symmetry, semicircular transitions are again predicted for specific combinations of conductivities in the cases of widely separated layers and for bilayers with large tunneling energies.

(4) Selection rules. We show how the experimentally successful selection rule, $p q^{\prime}-p^{\prime} q= \pm 1$, for monolayer transitions between plateaux characterized by Hall conductivities $p / q$ and $p^{\prime} / q^{\prime}$ (in units of $e^{2} / h$ ), generalizes to bilayer systems. For bilayers precisely the same selection rule holds (for particular combinations of conductivities) for all three categories of the flow mentioned above.

(5) Unresolved layers. It is often the case that the separate conductivities are not measured independently for bilayer systems, and we show in this case how the above predictions reduce to the case where only the total conductivity is measured. This allows the predictions of the symmetry to be applied more directly to single-layer systems for which it is the electron spins (or other labels) which distinguish the two 'layers'. The predictions in this case include the existence of even-denominator states and the selection rule becomes $p q^{\prime}-p^{\prime} q= \pm s$, where $s=1$ or 2 , depending on which of the three categories of flow is under consideration. For systems with particle-hole symmetry there should be semicircular transitions between these states.

\section{EMERGENT SYMMETRY OF THE LOW-ENERGY THEORY}

In this section we briefly describe the properties of the emergent symmetry in the bosonic and fermionic cases, starting first with a restatement of what is known for a single conducting fluid (as appropriate to a spin-split Landau level in a monolayer sample) and then generalizing to the case of two fluids (as would apply to a spin-degenerate layer or a bilayer consisting of two spin-split quantum Hall systems).

\section{A. Single-layer case}

It is the case of a spin-split single Landau level which has been longest studied, and we follow here the description of Ref. 10. In this reference it is argued that there are two operations which commute with the scaling flow in the lowenergy limit of any system in two spatial dimensions for which the quasiparticle charge carriers relevant to transport measurements are weakly coupled particles or vortices. These two generators do not commute with one another and their repeated application generates a large discrete group which resides within $\operatorname{SL}(2, Z)$.

In two dimensions particle statistics is described by an angle, $\varphi$, which represents the phase, $e^{i \varphi}$, which the particles acquire if they are adiabatically moved around one another. The first of the symmetry operations which must commute with the flow is simply the addition of $2 \pi$ statistics flux to the relevant charge-carrying quasiparticles, since this does not change at all the accumulated phase.

The second symmetry generator arises when the quasiparticles relevant for transport in some of the system's phases are weakly coupled vortices rather than charges. In this case the similar kinematics of charges and vortices in two dimensions implies the flow commutes with the operation of particle-vortex interchange.

Reference 10 shows that these two operations have a very simple action on the electromagnetic response of the twodimensional system, which for conductors and semiconductors acts on the complex conductivities $\sigma \equiv \sigma_{x y}+i \sigma_{x x}$ as a fractional linear transformation as follows:

$$
\sigma \rightarrow \frac{a \sigma+b}{c \sigma+d}
$$

where the integers $a, b, c$, and $d$ satisfy $a d-b c=1$. Because this is invariant under a change of sign for the constants $a-d$, this defines the group $\operatorname{PSL}(2, Z)$. It is convenient to express the transformations of this type in terms of two standard ones: 


$$
S(\sigma)=-\frac{1}{\sigma} \quad \text { and } \quad T(\sigma)=\sigma+1,
$$

which satisfy the identity $(S T)^{3}=1$. For instance, the implications for the conductivities of the addition of $2 \pi$ statistics flux to the charge carriers can be written in terms of these generators as the combination $S T^{2} S$, or $\sigma \rightarrow \sigma^{\prime}$, with

$$
\frac{1}{\sigma^{\prime}}=\frac{1}{\sigma}-2
$$

This transformation was derived by Jain and co-workers, ${ }^{15}$ although only in the context of $\sigma_{x y}$ and not on the full upperhalf complex plane.

The modular group has fixed points, in the sense that there is a special set of points $\sigma_{*}$ for which there exists an element $\gamma$ of the modular group that leaves $\sigma_{*}$ invariant, $\gamma\left(\sigma_{*}\right)=\sigma_{*}$. Quantum Hall plateaux are examples of such fixed points lying on the real axis, $\sigma_{*, x x}=0$, with $\sigma_{*, x y}=p / q$ a rational number (the requirement that $q$ be odd for semiconductors restricts the modular group to a subgroup as described in Sec. II A 2 below).

It is the specific values taken by the integers $a-d$ for the particle-vortex interchange which differs in the bosonic and fermionic cases, so we consider each of these cases separately after first discussing the implications of this symmetry for the scaling flow.

\section{Scaling flow}

The requirement that the scaling flow of the quantum Hall effect commutes with this infinite discrete group ${ }^{16}$ strongly restricts the general form of the flow in quantum Hall systems. The statement that the symmetry commutes with the flow means that the scaling function, the logarithmic derivative of the conductivity along a flow line $\mathcal{F}(\sigma, \bar{\sigma})=\frac{d \sigma}{d s}$ where $s$ is the logarithm of the scale, is the same regardless of whether the modular group is applied before or after taking the derivative, i.e.,

$$
\mathcal{F}(\sigma, \bar{\sigma})=\frac{d \sigma}{d s} \Leftrightarrow \mathcal{F}(\gamma(\sigma), \gamma(\bar{\sigma}))=\frac{d \gamma(\sigma)}{d s} .
$$

This proves to be a very strong restriction and has the consequence that fixed points of the modular symmetry must necessarily be fixed points of the scaling flow, i.e., if $\sigma_{*}$ is such that there exists an element $\gamma \in \Gamma_{0}(2)$ which leaves $\sigma_{*}$ invariant, $\gamma\left(\sigma_{*}\right)=\sigma_{*}$, then $\sigma_{*}$ is necessarily a fixed point of the scaling flow and $\mathcal{F}\left(\sigma_{*}, \bar{\sigma}_{*}\right)=0 .{ }^{16}$

The original scaling arguments of Khmel'nitskii ${ }^{17}$ and Pruisken ${ }^{18}$ were obtained from RG techniques, using an "effective" sample size $L$. In general the conductivity would be expected to be a function of a number of parameters, such as the magnetic field, the temperature, the charge carrier density, the impurity density, and the size. If the charge carrier and impurity densities are fixed then we have $\sigma(B, T, L)$, but simple scaling says that $\sigma$ is dimensionless (in units with $\left.e^{2} / h=1\right)$ so it only depends on two independent arguments and can be written as $\sigma\left(B / L^{\eta}, T / L^{\rho}\right)$ for some pair of exponents $\eta$ and $\rho$, or alternatively as $\sigma\left(B / T^{\eta^{\prime}}, L / T^{\rho^{\prime}}\right)$ for a dif-

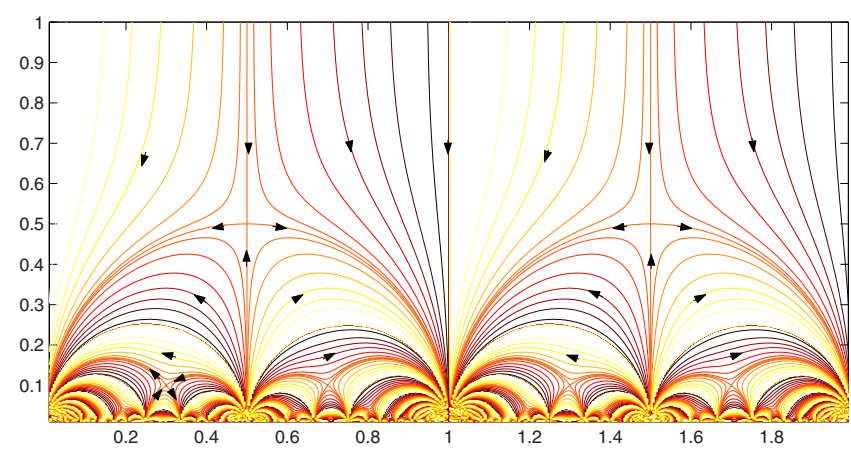

FIG. 1. (Color online) Temperature flow lines for the $\Gamma_{0}(2)$-invariant flow of $\sigma . \sigma_{x y}$ is plotted along the horizontal axis and $\sigma_{x x}$ up the vertical axis, in units with $e^{2} / h=1$. The arrows on the figure indicate the flow direction for decreasing temperature, assuming the samples behave as monolayer semiconductors when $B=0$.

ferent pair $\eta^{\prime}$ and $\rho^{\prime}$. One expects a different flow depending on whether the effective sample size $L$ is varied, $\mathcal{F}_{L}:=L \frac{d \sigma}{d L}$ $=-\left.\eta B \frac{\partial \sigma}{\partial B}\right|_{T}-\left.\rho T \frac{\partial \sigma}{\partial T}\right|_{B}$, or the temperature $T$ is varied, $\mathcal{F}_{T}$ $:=T \frac{d \sigma}{d T}=-\left.\eta^{\prime} B \frac{\partial \sigma}{\partial B}\right|_{L}-\left.\rho^{\prime} L \frac{\partial \sigma}{\partial L}\right|_{B}$. If $B$ is held fixed then $\mathcal{F}_{L}=$ $-\rho T \frac{\partial \sigma}{\partial T}$ and $\mathcal{F}_{T}=-\rho^{\prime} L \frac{\partial \sigma}{\partial L}$.

The experiments of Murzin et al. ${ }^{19}$ are in remarkable agreement with Fig. 1 above and indicate that it is reasonable to consider the flows obtained from modular symmetry for semiconductors as arising from $\mathcal{F}_{T}$.

Anticipating the discussion of fermions and considering Fig. 1 above, where an example of the flow dictated by modular symmetry is shown, we note that the topology of the flow does not require full knowledge of the functional form of $\mathcal{F}$. If Fig. 1 is taken to represent $\mathcal{F}_{L}$ one can multiply $\mathcal{F}_{L}$ by any function of $L$ which is nonvanishing away from the critical points and the topology of the figure does not change. Alternatively, if Fig. 1 represents $\mathcal{F}_{T}$ one can multiply $\mathcal{F}_{T}$ by any function of $T$ which is nonvanishing away from the critical points and the topology of the figure does not change. These two alternative views are quite compatible, indeed Fig. 1 could represent both $\mathcal{F}_{L}$ and $\mathcal{F}_{T}$, if their ratio is a nonvanishing real function of $T$ and $L$, and this is perhaps not unreasonable from a field theoretical point of view in $2+1$ dimensions. $L$ can be taken to be the size of the system, e.g., the spatial extent of the system at low $T$, while in field theory $1 / T$ would be the temporal extent of the system, so the distinction between the two functions is that of temporal versus spatial variations.

In the following we shall take the pragmatic attitude, given the experimental data, that the flows represent $\mathcal{F}_{T}$.

\section{Fermionic symmetry}

If the charge carriers for one of the system's phases are fermions (or related to fermions by a symmetry transformation), then the implication for the conductivities of interchanging a particle with a vortex is $\sigma \rightarrow \sigma^{\prime}$ with

$$
\sigma^{\prime}=\frac{\sigma-1}{2 \sigma-1} .
$$

The group generated by iterating this transition with that of Eq. (4) consists of all $\operatorname{PSL}(2, Z)$ transformations for which $c$ 
is even, which defines the subgroup $\Gamma_{0}(2)$. This group is generated by the two generators $T$ and $S T^{2} S$.

When supplemented with a symmetry interchanging particles and holes (i.e., $\sigma \rightarrow 1-\bar{\sigma}$ ) completely determines a particular class of semicircular flow lines. ${ }^{8}$ Combining this with a boundary condition at high temperatures (or vanishing magnetic field, etc.) to fix the overall direction of flow leads to the desired observational consequences.

For instance, assuming the flow direction at large $\sigma_{x x}$ (or at $\sigma_{x y}=0$ ) corresponds to that of a semiconducting monolayer sample leads to the temperature flow shown in Fig. 1 (which is a more detailed version of Fig. 1 in the first reference of Ref. 8), where the arrows indicate the direction of decreasing temperature and different flow lines correspond to different values of $B$. As the temperature is lowered the flow from above is forced onto semicircles of unit diameter spanning the integers on the real line and, at low enough temperatures, these semicircles are followed when $B$ is varied to force transitions between integer Hall plateaux. This pattern is repeated in a self-similar pattern for transitions between rational fractions.

There are completely attractive fixed points on the real line at rational fractions $\sigma=p / q$ with $q$ odd (these are images of $\sigma=1$ under the group action) and completely repulsive fixed points at even denominators, $\sigma=p / q$ with $q$ even (these are images of $\sigma=i \infty)$. There are saddle points, attractive in one direction and repulsive in another, at $\sigma=(1+i) / 2$ and its images - these represent critical points on the transition between two Hall plateaux as the magnetic field is varied.

In the mathematical limit of perfect modular symmetry there is a fractal structure just above the real line, but in any real sample there is obviously a maximum value for the denominator of the filling fraction-there can be no truly infinite fractals in nature-though purer samples with higher mobilities can achieve higher denominators.

Among the consequences of this flow are the following universal properties:

$M 1$. The flow as temperatures go to zero is toward the attractive fixed points, the complete list of which is $\sigma=p / q$ with $q$ odd.

$M 2$. The plateaux, $\sigma=p / q$ and $\sigma^{\prime}=p^{\prime} / q^{\prime}$, which may be connected by a semicircle must satisfy the selection rule $p q^{\prime}-p^{\prime} q= \pm 1$.

$M 3$. If particle-hole interchange is also a symmetry, in the sense of creating a hole when a particle is removed from a full Landau level $(\sigma \rightarrow 1-\bar{\sigma})$, then at low temperatures the flow between plateaux obtained as magnetic fields are varied traverses a semicircle in the complex conductivity plane, with the semicircle centered on the real axis. ${ }^{8}$ This includes also vertical lines, which may be regarded as infinitely large semicircles, leading to the curves shown in Fig. 1.

These describe very well the observed transitions which are seen in the spin-split single-layer quantum Hall systems in semiconducting heterojunctions.

\section{Bosonic symmetry}

On the other hand, if the charge carriers for one of the system's phases should be bosons (or related to them by a symmetry transformation), then the group generated by iter- ating particle-vortex interchange with $2 \pi$ statistics flux addition consists of all those $\operatorname{PSL}(2, Z)$ transformations for which both $b$ and $c$ are even, or for which both are odd. This defines the subgroup $\Gamma_{\theta}$, which is generated by the two generators $S$ and $S T^{2} S$ (or equivalently $S$ and $T^{2}$ ). This symmetry was first suggested in connection with the quantum Hall effect in Ref. 6.

The requirement that the flow commutes with this group dictates its observational consequences. ${ }^{8}$ These include the last two consequences listed above for fermionic systems, but the first consequence is instead changed to the following:

$M 1^{\prime}$. The flow as temperatures go to zero is toward the attractive fixed points, the complete list of which is $\sigma=p / q$, where $p$ and $q$ are relatively prime and the product $p q$ must be even.

Notice in this case that the selection rule $M 2$ requires transitions to be between plateaux for which $p / q$ =even/odd and those for which $p / q=$ odd/even.

Because the fixed points differ between the cases of bosons and fermions, the precise properties of the semicircles predicted in the bosonic and fermionic cases differ in detail. Pictures of the allowed semicircles in both cases are given in Ref. 10. Unlike for the fermionic case, experimental systems exhibiting these predictions were not known, although the recent observations of quantum Hall phenomena in graphene may provide the first example. ${ }^{21}$

\section{B. Double-layer case}

For quantum Hall bilayers there are more conductivities which are accessible to observations, since there are Ohmic and Hall conductivities for each layer separately, $\sigma_{11}$ and $\sigma_{22}$, as well as the interlayer conductivities, $\sigma_{12}=\sigma_{21}$. Here the labels "1" and "2" distinguish the two layers, and each of the $\sigma_{i j}$ are complex numbers, whose real part gives the corresponding Hall conductivity and whose imaginary part gives the Ohmic conductivity.

Our interest is to generalize the discrete symmetry of the single-layer case to the bilayer context, and it is convenient for this purpose to group these complex conductivities into a complex matrix

$$
\Sigma=\left(\begin{array}{ll}
\sigma_{11} & \sigma_{12} \\
\sigma_{21} & \sigma_{22}
\end{array}\right) .
$$

In this case we know that the flow must commute with the action of the discrete symmetry when it is applied to each layer separately. If the layers are identical we may also demand that the flow commute with the symmetry corresponding to the interchange of the two layers. This leads us to expect there to be a total of five independent symmetry generators in the bilayer case.

In terms of the matrix $\Sigma$ these transformations form a subgroup of the discrete group

$$
\Sigma \rightarrow(A \Sigma+B)(C \Sigma+D)^{-1},
$$

where the $2 \times 2$ matrices $A, B, C$, and $D$ have integer entries. Denoting the transpose by the superscript $t$, these matrices must satisfy the conditions $A B^{t}=B A^{t}, C D^{t}=D C^{t}$, and $A D^{t}$ $-B C^{t}=1$. The four matrices $A, B, C$, and $D$, with this con- 
straint, represent an element $\gamma=\left(\begin{array}{ll}A & B \\ C & D\end{array}\right)$ of the group $\operatorname{Sp}(4, Z)$. A mathematical property of this transformation that is important for the physics of the quantum Hall effect in bilayers is that, when the imaginary part of $\Sigma$ is positive (i.e., its eigenvalues are positive) then Eq. (8) preserves this property.

Since the precise subgroup defined by the particle-vortex symmetry for each layer depends on whether the pseudoparticle charge carriers are related by the symmetry to bosons or fermions, the same is also true for the bilayer case. We must ask for the explicit form for the matrices $A-D$ separately for the case of bosonic and fermionic systems.

\section{Fermionic symmetry}

For bilayers built from two fermionic layers the five symmetry generators can be taken to be given by the following transformations, which express the symmetry $\Gamma_{0}(2)$ acting separately on each layer, together with interlayer interchange, $P$. Collecting the matrices $A-D$ into a $4 \times 4$ matrix

$$
\gamma \equiv\left(\begin{array}{ll}
A & B \\
C & D
\end{array}\right)
$$

the five generators are

$$
\begin{gathered}
T_{1}=\left(\begin{array}{cc}
1 & \frac{1}{2}\left(1+\tau_{3}\right) \\
0 & 1
\end{array}\right), \\
S_{1} T_{1}^{2} S_{1}=\left(\begin{array}{cc}
-\tau_{3} & 0 \\
1+\tau_{3} & -\tau_{3}
\end{array}\right), \\
T_{2}=\left(\begin{array}{cc}
1 & \frac{1}{2}\left(1-\tau_{3}\right) \\
0 & 1
\end{array}\right), \\
S_{2} T_{2}^{2} S_{2}=\left(\begin{array}{cc}
\tau_{3} & 0 \\
1-\tau_{3} & \tau_{3}
\end{array}\right),
\end{gathered}
$$

and

$$
P=\left(\begin{array}{cc}
\tau_{1} & 0 \\
0 & \tau_{1}
\end{array}\right) .
$$

Here $\tau_{k}$ denotes the usual $2 \times 2$ Pauli matrices, with

$$
\tau_{1}=\left(\begin{array}{ll}
0 & 1 \\
1 & 0
\end{array}\right) \text { and } \tau_{3}=\left(\begin{array}{cc}
1 & 0 \\
0 & -1
\end{array}\right) .
$$

Most of the remainder of this paper is devoted to the exploration of the observational consequences of this symmetry.

\section{Bosonic symmetry}

Before exploring the consequences of the fermionic bilayer symmetry, we pause briefly to record the symmetry which would apply to bilayers built from two bosonic layers. In this case the five symmetry generators can instead be found by replacing the generators $T_{1}$ and $T_{2}$ by the following two generators:

$$
\begin{aligned}
& S_{1}=\left(\begin{array}{cc}
\frac{1}{2}\left(1-\tau_{3}\right) & \frac{1}{2}\left(1+\tau_{3}\right) \\
-\frac{1}{2}\left(1+\tau_{3}\right) & \frac{1}{2}\left(1-\tau_{3}\right)
\end{array}\right), \\
& S_{2}=\left(\begin{array}{cc}
\frac{1}{2}\left(1+\tau_{3}\right) & \frac{1}{2}\left(1-\tau_{3}\right) \\
-\frac{1}{2}\left(1-\tau_{3}\right) & \frac{1}{2}\left(1+\tau_{3}\right)
\end{array}\right) .
\end{aligned}
$$

For simplicity we here do not further explore the consequences of this group.

\section{Identical layers}

In order to explore the consequences of the fermionic bilayer symmetry, $G$, it is useful to specialize to the experimentally relevant case where both of the layers are identical. In this case we may write the intralayer conductivities as $\sigma_{11}=\sigma_{22} \equiv \sigma$, and the interlayer conductivities as $\sigma_{12}=\sigma_{21}$ $\equiv \widetilde{\sigma}$, and so

$$
\Sigma=\left(\begin{array}{cc}
\sigma & \widetilde{\sigma} \\
\widetilde{\sigma} & \sigma
\end{array}\right)=\sigma \tau_{0}+\widetilde{\sigma} \tau_{1},
$$

where $\tau_{0}=I$ denotes the $2 \times 2$ unit matrix. With this choice the generator $P$ acts trivially on $\Sigma$.

In this case the implications of the remaining four generators of $G$ are more conveniently identified by following its action on the following combinations: $\sigma_{ \pm}:=\sigma \pm \widetilde{\sigma}$, in terms of which we have

$$
\Sigma=\sigma_{+} \tau_{+}+\sigma_{-} \tau_{-},
$$

where $\tau_{ \pm}$denote the projection matrices $\tau_{ \pm}=\frac{1}{2}\left(\tau_{0} \pm \tau_{1}\right)$. Physically, the conductivities $\sigma_{ \pm}$describe correlations of the currents $J_{ \pm}=J_{1} \pm J_{2}$, where $J_{i}$ denotes the electrical current passing through layer $i$.

The action of $G$ on these variables is most simply expressed by also focusing on the matrices $A-D$ which can also be written in terms of $\tau_{ \pm}: A=a_{+} \tau_{+}+a_{-} \tau_{-}$, and so on. The integers $a_{ \pm}, b_{ \pm}, c_{ \pm}$, and $d_{ \pm}$obtained in this way must satisfy $a_{n} d_{n}-b_{n} c_{n}=1$ separately for $n=+$ and $n=-$. Similarly, the matrix $C$ obtained in this way is even provided the constants $c_{ \pm}$are also even.

Using these matrices it is clear that the action of $G$ on $\Sigma$ decomposes into a separate $\Gamma_{0}(2) \subset \operatorname{SL}(2, Z)$ action on each of $\sigma_{ \pm}$- denoted $\Gamma_{0}^{ \pm}(2)$ - with

$$
\sigma_{n} \rightarrow \frac{a_{n} \sigma_{n}+b_{n}}{c_{n} \sigma_{n}+d_{n}}, \quad n= \pm
$$

It is also clear that these two copies of $\Gamma_{0}(2)$ commute with one another.

The advantage of writing things this way is that it allows the results of Ref. 10 to be taken over in whole cloth, since this action is mathematically identical to the action of the fermionic symmetry on a monolayer. In particular, we see that writing the symmetry in this way implies that as the 
temperature is varied, the topology of the flow is given as in Ref. 10 . There are then potentially two qualitatively different flow patterns, which differ only in the direction of the flow along the lines drawn in Fig. 1.

When the flow with decreasing temperature is in the same direction as is shown in Fig. 1 then an initially large Ohmic conductivity falls with temperature, as is the case for the semiconducting monolayer systems. Continuity implies that this direction of flow also holds in the limit of bilayers which consist of two monolayers which are sufficiently far apart from one another. This need no longer be true if a transition should occur to a qualitatively different ground state at a critical separation, such as happens once interlayer correlations become important.

The curves followed when magnetic field, $B$, (or carrier density, $n$ ) are varied at low temperatures can also be inferred from temperature-flow diagrams like Fig. 1. For these purposes notice that different vertical lines correspond to different initial magnetic fields, and (given the direction of flow given in Fig. 1) if these start from the top of the figure (as is true for comparatively low-mobility samples) then they are attracted to integer plateaux (such as those at $\sigma=0$ and $\sigma$ $=1$ in Fig. 1) as $T \rightarrow 0$. By comparing the position for differing magnetic fields at a common low temperature one can see that the trajectories followed as $B$ is varied for fixed $T$ near zero lie along the separatrix defined by the semicircle connecting $\sigma=0$ to $\sigma=1$ in Fig. $1 .{ }^{10}$ In this case the existence of the symmetry implies the following generalizations of the monolayer predictions to bilayer systems.

$B 1$. The flow as temperatures go to zero is toward the attractive fixed points, the complete list of which is $\sigma_{ \pm}$ $=p_{ \pm} / q_{ \pm}$with $q_{ \pm}$odd.

$B 2$. The plateaux, $\sigma_{ \pm}=p_{ \pm} / q_{ \pm}$and $\sigma^{\prime}=p_{ \pm}^{\prime} / q_{ \pm}^{\prime}$, which may be related by such a semicircle must satisfy the selection rule $p_{n} q_{n}^{\prime}-p_{n}^{\prime} q_{n}= \pm 1$ separately for $n=+$ and $n=-$.

$B 3$. At low temperatures (if particle-hole interchange is a symmetry) the flow between plateaux obtained as the magnetic fields are varied traverses a semicircle in the complex $\sigma_{+}$and a semicircle the complex $\sigma_{-}$plane, with the semicircles centered on the real axis in each case. This last prediction assumes the two plateaux in question lie in the same superuniversality class-an exception to this semicircle rule arises if the varying magnetic field triggers a change in the category of the super-universality class of the transitions (as we discuss in more detail below).

On the other hand, bilayers states have been seen which exhibit properties more usually associated with superconductors rather than semiconductors and for these states an initially large conductivity would be expected to get larger as the temperatures drops, rather than lower as in a semiconductor. For these states the flow for decreasing temperature is again along the lines of Fig. 1, but with the direction of the flow lines reversed from those drawn in the figure. The existence of such a flow is consistent with the symmetry, and cannot be excluded on continuity grounds if the bilayer system in question is separated from the limit of well-separated layers by a discontinuous transition.

Indeed, we shall argue below that real bilayer systems do exhibit low-temperature fixed points of this new form, including examples ${ }^{23,24}$ for which $\sigma_{+}=\frac{1}{4}$ and others ${ }^{25}$ for which the resistivity matrix is given by

$$
-\Sigma^{-1}=\left(\begin{array}{ll}
1 & 1 \\
1 & 1
\end{array}\right)
$$

These states, which arise for small interlayer tunneling energies, are believed to involve a superfluid condensation of the current corresponding to opposite-charge flow in the two layers, and so are necessarily separated from the limit of widely separated layers by a phase transition. We return to a more detailed discussion of the phenomenology of these new flows below.

For any such a phase having reversed flow direction, repeating the arguments of Ref. 10 leads instead to the following predictions:

$B 1^{\prime}$. The flow as temperatures go to zero is toward the attractive fixed points, the complete list of which is $\sigma_{ \pm}$ $=p_{ \pm} / q_{ \pm}$with $p_{ \pm}$odd and $q_{ \pm}$even.

$B 2^{\prime}$. The plateaux, $\sigma_{ \pm}=p_{ \pm} / q_{ \pm}$and $\sigma^{\prime}=p_{ \pm}^{\prime} / q_{ \pm}^{\prime}$, which may be related by such a trajectory must satisfy the selection rule $p_{n} q_{n}^{\prime}-p_{n}^{\prime} q_{n}= \pm 2$ separately for $n=+$ and $n=-$.

$B 3^{\prime}$. At low temperatures, if particle-hole interchange is a symmetry, the flow between plateaux obtained as the magnetic field is varied traverses a semicircle in the complex $\sigma_{+}$ and a semicircle in the complex $\sigma_{-}$plane.

As discussed above, both of the latter two predictions require there to be no discontinuous transition as $B$ is varied which could change the category of superuniversality class of symmetries that is relevant.

We see in this way the possibility of two different categories of transitions amongst bilayer systems (and two more if $\Gamma_{\theta}$ should also be a relevant group), with each being governed by a qualitatively different kind of flow. In both cases the trajectories of the conductivities have the shape given by Fig. 1, but the two categories differ in the direction of this flow as the temperature falls. The above predictions apply to the transitions between phases, provided that the phases involved both belong to the same category.

\section{Two layers unresolved}

More often than not experiments do not separately distinguish the currents and voltages in any one layer, and quote only a partial measurement corresponding to the total current and voltage across both layers. This is the case both for bona fide bilayer systems as well as for spin-degenerate monolayers for which it is electron spin which plays the role of layer number. Because these types of measurements are so common, we pause here to state what the above symmetry statements become in this important special case.

If the layers (or spins) are not distinguished observationally, then all that is measured is the total conductivity, $\sigma_{T}$, which is related to the inter- and intralayer conductivities by $\sigma_{T}=\sigma_{11}+\sigma_{22}+\sigma_{12}+\sigma_{21}$. In the case of identical layers this simplifies to

$$
\sigma_{T}=2(\sigma+\widetilde{\sigma})=2 \sigma_{+} .
$$

Since we have seen that the bilayer symmetry generates a $\Gamma_{0}(2)$ action of $\sigma_{+}$onto itself, we see that it also must generate an action on $\sigma_{T}$. Interestingly, because of the factor of 2 in the relationship between $\sigma_{T}$ and $\sigma_{+}$, the action generated 
on $\sigma_{T}$ is not simply $\Gamma_{0}(2)$. Instead, it is the transformation $\sigma_{T} \rightarrow\left(a \sigma_{T}+b\right) /\left(c \sigma_{T}+d\right)$ with $b$ even, which defines the group $\Gamma^{0}(2) \subset \operatorname{SL}(2, Z) .{ }^{27}$ This can be seen because $\Gamma^{0}(2)$ is generated by $T^{2}$ and STS and $\sigma_{T} \rightarrow \sigma_{T}+2$ and $\sigma_{T} \rightarrow \sigma_{T} /(1$ $\left.-\sigma_{T}\right)$ are clearly equivalent to $\sigma_{+} \rightarrow \sigma_{+}+1$ and $\sigma_{+} \rightarrow \sigma_{+} /(1$ $\left.-2 \sigma_{+}\right)$when $\sigma_{T}=2 \sigma_{+}$.

We see that the total conductivity, $\sigma_{T}$, of unresolved bilayer systems enjoys a $\Gamma^{0}(2)$ symmetry, which has slightly different consequences than does $\Gamma_{0}(2)$. There are again two cases, depending on the overall direction of the flow as the temperature decreases. In particular, if the flow lines are the same as for a semiconducting monolayer, predictions $B 1-B 3$ for $\sigma_{+}$imply the following for $\sigma_{T}$ :

$S 1$. The flow as temperatures go to zero is toward the attractive fixed points, the complete list of which is $\sigma_{T}$ $=p / q$ with $p$ even and $q$ odd.

$S 2$. The plateaux, $\sigma_{T}=p / q$ and $\sigma^{\prime}=p^{\prime} / q^{\prime}$, which may be related by varying magnetic fields at low temperatures must satisfy the selection rule $p q^{\prime}-p^{\prime} q= \pm 2$.

$S 3$. For samples exhibiting particle-hole symmetry the flow between plateaux obtained by varying the magnetic field at low temperature traverses a semicircle in the complex $\sigma_{T}$ plane, with the semicircle centered on the real axis.

As usual the latter two of these predictions require there is no discontinuous change of ground state as the transition is made. In particular, continuity implies that these properties hold in the limit of widely separated monolayers, for which interlayer coherence and correlations are negligible.

On the other hand, for transitions within the category for which the direction of the flow lines is reversed compared with Fig. 1 we have instead the predictions $B 1^{\prime}-B 3^{\prime}$ for $\sigma_{+}$, which in the present context become

$S 1^{\prime}$. The flow as temperatures go to zero is toward the attractive fixed points, the complete list of which is $\sigma_{T}$ $=p / q$ with $p$ odd.

$S 2^{\prime}$. The plateaux, $\sigma_{T}=p / q$ and $\sigma_{T}^{\prime}=p^{\prime} / q^{\prime}$, which may be related at low temperatures by varying the magnetic field must satisfy the selection rule $p q^{\prime}-p^{\prime} q= \pm 1 .^{28}$

$S 3^{\prime}$. For systems with particle-hole symmetry the trajectory traversed when varying $B$ at low temperatures follows a semicircle centered on the real axis.

\section{E. Transitions with varying $B$}

We see that, when particle-hole symmetry is present, the condition that the flow commutes with the bilayer symmetry predicts a generalized semicircle law for the trajectories which the conductivities take at low temperatures as $B$ is varied. However, since changes in $B$ can also drive transitions between the different categories of phases of the bilayer systems, it is important to keep in mind that the semicircular trajectories need not follow if the system undergoes a transition from one category of phases to another. In order to understand the implications of the above predictions for real systems we must therefore keep track of how the phase of the system changes as the magnetic field $B$ (or magnetic length, $\left.\ell \propto B^{-1 / 2}\right)$ varies.

\section{Relevant energy scales}

Physically, we expect that the kinds of phases which are possible for a given system depend on the relative strength of

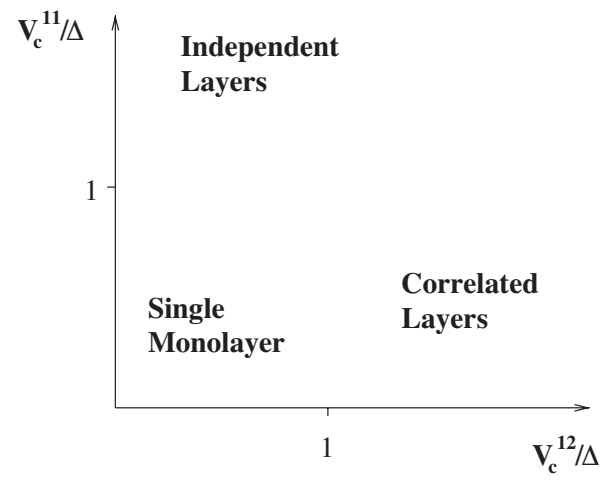

FIG. 2. A sketch of the phase diagram describing the three regimes described in the text as functions of intralayer and interlayer Coulomb correlation energies normalized to the tunneling energy, $\Delta$.

a typical interlayer tunneling matrix energy, $\Delta$, compared with the inter- and intraplane Coulomb correlation energies: $V_{c}^{12}$ and $V_{c}^{11}$. In terms of these quantities we expect the following three regimes, which are schematically pictured as a "phase diagram" in Fig. 2:

(1) When $\Delta$ dominates both $V_{c}^{11}$ and $V_{c}^{12}$ then interlayer tunneling is large and the number of electrons in each layer is not separately conserved. In this case the Fermi level can fall between the energies of the states whose wave functions are symmetric and antisymmetric under interlayer interchange, leading to what is effectively a single-layer system, denoted 'single monolayer' in Fig. 2. If $\Gamma_{0}(2)$ is the relevant group then the older predictions, $M 1-M 3$ or $M 1^{\prime}-M 3^{\prime}$, given above which apply. This would be expected to be the case at small $B$.

(2) If $V_{c}^{11}$ is sufficiently dominant over $V_{c}^{12}$ and $\Delta$ then we expect the system to behave as two independent layers, denoted 'independent layers' in the figure. In this case we expect the direction of the temperature flow to be the same as for each monolayer separately, and if $\Gamma_{0}(2)$ is the relevant group then it is the predictions $B 1-B 3$ above which apply. (If the conductivities in each layer are not distinguished, then it is instead the predictions $S 1-S 3$ which would be relevant.)

(3) When $V_{c}^{12}$ and $V_{c}^{11}$ are both more important than $\Delta$, then interlayer Coulomb correlations play a role and we expect new bilayer plateaux to appear, denoted 'correlated layers' in Fig. 2. This includes the situation of Bose-Einstein condensation among the exciton states which form from pairs of electrons and holes in different layers. In this situation the existence of the superfluid state leads us to expect the temperature flow to be opposite to that of the decoupled monolayers, because conductivity decreases in a semiconductor as the temperature is lowered and increases in a superconductor. This leads to the expectation that predictions $S 1^{\prime}-S 3^{\prime}$ are the relevant ones.

So far this entire discussion applies just as well to spindegenerate single-layer QHE systems as it does to spin-split bilayers, provided that we identify $\Delta$ as the Zeeman-splitting matrix element which is responsible for the non-Coulomb part of the energy difference between the differing spin states. 


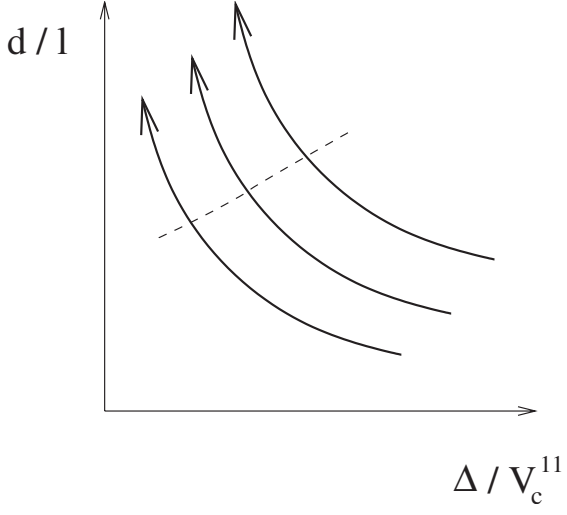

FIG. 3. An illustration of the relative strengths of the intralayer Coulomb energy and $\Delta$ compared to the dimensionless ratio $d / l$, taken from. Ref. 20. The curves correspond to increasing $B$ at constant charge carrier density and the correlated bilayer state is only realized below the dotted line.

\section{Magnetic field dependence}

Because the energies $\Delta, V_{c}^{11}$, and $V_{c}^{12}$ all depend differently on the magnetic field strength, any particular sample traverses a trajectory in the phase diagram of Fig. 2 as $B$ is varied. It is here that we must distinguish between bilayer and spin-degenerate monolayer systems, because they differ in the $B$ dependence which is likely for these energy scales. This difference has important implications for the kinds of trajectories which are possible in Fig. 2 for each type of system as the magnetic field is varied.

Bilayer systems. For bilayers $\Delta$ is not expected to depend strongly on $B$, but it does depend strongly on the interlayer distance $d$, falling to zero exponentially quickly as the layers are separated. The way in which the phase structure can depend on magnetic field was examined in Ref. 20 and Fig. 3 is a sketch of Fig. 1 from that paper. That analysis indicates that, at large $B$, the ratio of the two length scales $d$ and the magnetic length $l \propto 1 / \sqrt{B}$ determines whether or not the system behaves as two independent monolayers or as a correlated bilayer, as shown in Fig. 3 above. As the magnetic field increases, at constant charge carrier density, the importance of the intralayer Coulomb energy $\left(V_{c}^{11} \propto 1 / l \propto \sqrt{B}\right)$ relative to $\Delta$ increases until intralayer Coulomb interactions destroy the correlated state and the system behaves as independent bilayers, provided $V_{c}^{11} \gg V_{c}^{12} \sim 1 / d$.

We see from these considerations that for semiconducting bilayers at small magnetic fields $\Delta$ should always dominate over Coulomb energies, implying a description in terms of a single monolayer, for which the predictions $M 1-M 3$ would be expected to hold. As $B$ increases the Coulomb correlation energies become more important and, if $V_{c}^{12}$ becomes important enough, a transition to the correlated bilayer regime can occur (for which predictions such as $B 1^{\prime}-B 3^{\prime}$ can apply), depending on the detailed properties of the sample in question. Eventually for large enough $B$ we expect $V_{c}^{11}$ to dominate, leading to the behavior of independent bilayers, for which we expect $B 1-B 3$ to be relevant.

As we shall see, this sequence of pictures seems to provide a good description of the kinds of phases which are seen

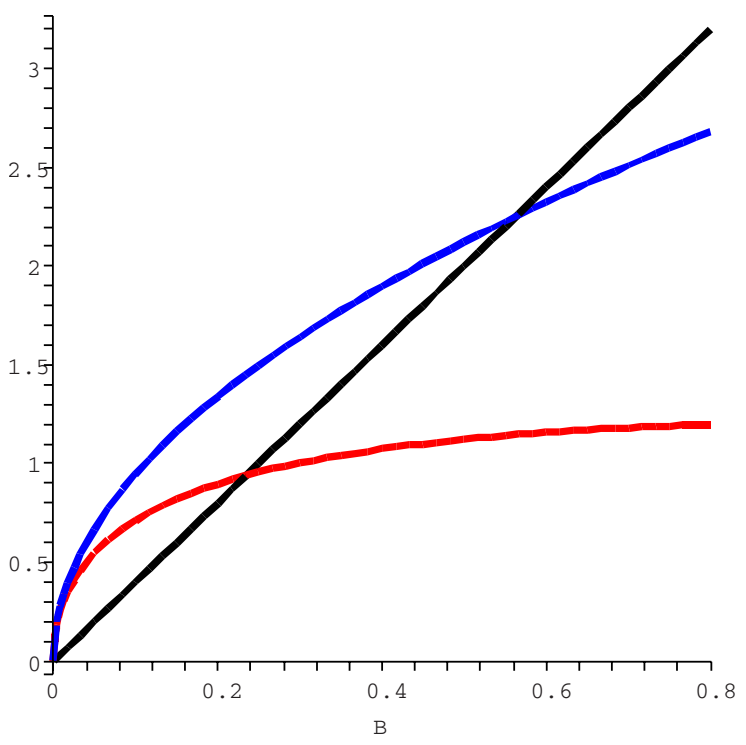

FIG. 4. (Color online) A sketch of the energies $V_{c}^{11}$ (upper) and $V_{c}^{12}$ (lower) for monolayer systems with spin-degenerate electrons. The straight line shows the Zeeman splitting, $\Delta$.

in real quantum Hall bilayers, although more detailed measurements to confirm or refute this picture would be most welcome.

Spin-degenerate monolayers. A similar discussion can be made for spin systems, with two provisos: (i) that the conductivities of the two spins of electron are not separately measured; and (ii) that some of the relevant energy scales depend differently on $B$ than they do for bilayer systems.

For spin systems the quantities $V_{c}^{12}$ and $V_{c}^{11}$ measure the spin-dependent matrix elements of the Coulomb interaction in a single plane, and so for the purposes of the qualitative estimates in this section we take both to be proportional to $1 / \ell \propto B^{1 / 2}$. For spin systems we also take the matrix element $\Delta$ to be due to the Zeeman interaction, and so $\Delta \propto B$.

Figure 4 gives a sketch of the relative size of these energies as functions of $B$. In the case sketched the small- $B$ regime is dominated by the Coulomb energies, assuming a sample with high enough mobility that Quantum Hall plateaux are visible at all. Assuming $\Gamma_{0}(2)$ is the relevant group, this leads to the predictions $S 1-S 3$ (or $S 1^{\prime}-S 3^{\prime}$ ), depending on the relative importance of $V_{c}^{11}$ and $V_{c}^{12} \cdot{ }^{29}$ For larger $B$ the energy $\Delta$ eventually dominates to the extent that our simple $B$ parametrization continues to hold, leading to a monolayer behavior, with predictions $M 1-M 3$.

\section{APPLICATIONS}

We now explore how the above observations can be used to make nontrivial predictions for real quantum Hall systems. What makes this tricky is the fact that when $B$ is varied, real systems can cross over between the different kinds of categories of phases (as described above) for which different symmetry predictions apply. Such transitions are problematic because we only know that the predictions hold for transitions amongst plateaux within any one of these categories, and not 


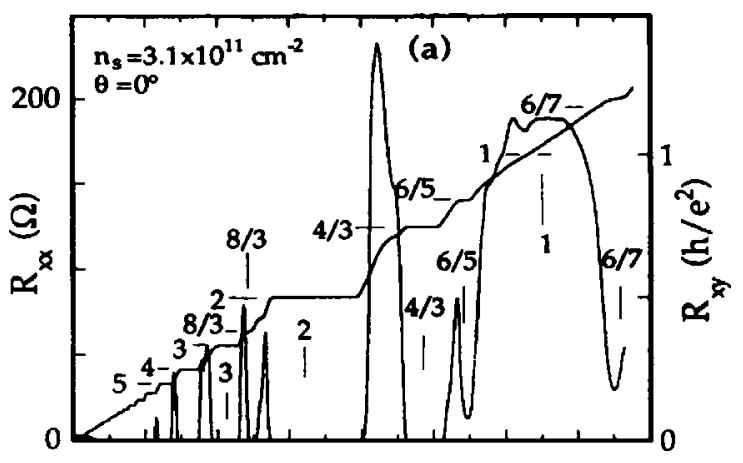

FIG. 5. Resistance traces for a bilayer system, reproduced from Fig. 2 of Suen et al. (Ref. 23). B increases to the right in this plot, with the same scale as for Fig. 7.

for transitions in between different categories.

In order to see how the above arguments may be used in practice, we now examine their implications in more detail for several bilayer systems for which experimental results are available in the literature. Our strategy for doing so relies on the fact that for each category described above there are three different nontrivial predictions: (i) those governing the kinds of plateaux allowed (e.g., $B 1, S 1, B 1^{\prime}$, or $S 1^{\prime}$ ), (ii) those governing the selection rules which express the plateaux that may be obtained from any given one by varying $B$ (e.g., $B 2$, $S 2, B 2^{\prime}$, or $S 2^{\prime}$ ), and (iii) those describing the shape of the trajectories which the conductivities take as $B$ is varied between these plateaux (e.g., $B 3, S 3, B 3^{\prime}$, and $S 3^{\prime}$ ). Our strategy is to use the kinds of plateaux to identify the category of phases that are present for each value of $B$. Once these categories are identified in this way we may then test the existence of the symmetry by asking whether the other two predictions hold for transitions within each category.

\section{A. Unresolved bilayers}

Most of the bilayer systems which have been studied experimentally fall into our "single-layer" category, for which only the total conductivity, $\sigma_{T}$, is measured. For the purposes of this section we take the example of Refs. 23 and 24, which together provided the first evidence for new kinds of Hall plateaux in these systems having $\sigma_{T}=\frac{1}{2}$. Both of these references provide explicit traces of the total Ohmic and Hall resistances at low temperatures as functions of magnetic field. A representative trace as given in Ref. 23 is reproduced here as Fig. 5.

At weak fields a series of integer plateaux are seen with $\sigma_{T}=5,4,3$ culminating in the first fractional state at $\sigma_{T}=\frac{8}{3}$. This is what is expected when tunneling dominates, including the appearance of the fractional state once the Coulomb interaction becomes large enough to compete with the others. Throughout this region we expect predictions $M 1-M 3$ to apply, including the selection rule $\left|p q^{\prime}-p^{\prime} q\right|=1$ (which can be seen from Fig. 5). The success of the picture can be tested on samples with particle-hole symmetry due to the prediction M3 of a semicircle law for these transitions, for which measurements would be most welcome.

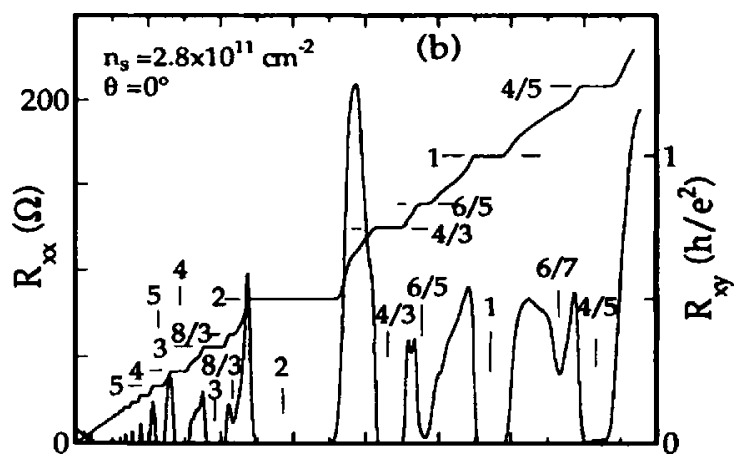

FIG. 6. Resistance traces, from Fig. 2 of Suen et al. (Ref. 23) for the same bilayer system as Fig. 5 but with smaller carrier density, $n_{s}$, and so smaller effective interlayer separation, $d$.

Although all of the plateaux visible in Fig. 5 have odd denominators, they cannot all be explained by the regime for which $\Delta$ dominates. Instead a transition occurs to the independent-bilayer category, for which predictions $S 1-S 3$ apply. The plateaux in Fig. 5 with $\nu>8 / 3$ do indeed have even numerators, but this in itself is not sufficient to distinguish between $M 1$ and $S 1$. That the transition to $S 1-S 2$ occurs is instead seen in Fig. 5 from the change to the selection rule $\left|p q^{\prime}-p^{\prime} q\right|=2$ for the plateaux above $\sigma_{T}=\frac{8}{3}$, indicating that $S 2$ applies rather than $M 2$. For particle-hole symmetric samples $S 3$ the prediction of a semicircle law again applies, but one which links plateaux obeying the new selection rule $(S 2)$ rather than the old one (M2).

Of all the visible plateaux the selection rule $\left|p q^{\prime}-p^{\prime} q\right|$ $=2$ only fails for the transition from $\sigma_{T}=\frac{6}{5}$ to $\sigma_{T}=\frac{6}{7}$, but there also appears to be considerable structure between these two plateaux (including the incipient state at $\sigma_{T}=1$-more about which below) which indicates unresolved intervening physics.

Figure 6 displays the plateaux found for the same sample but with a smaller carrier density, $n_{s}$ (obtained by applying a different bias voltage). As discussed in Ref. 23 this lower carrier density implies a lower effective interlayer separation, $d$, leading to nontrivial interlayer Coulomb correlations. For such a regime the predictions $S 1^{\prime}-S 3^{\prime}$ would apply, implying that the observed plateaux should have $\sigma_{T}=p / q$ with $p$ odd, as well as the reemergence of the selection rule $\mid p q^{\prime}$ $-p^{\prime} q \mid=1$. This picture appears to be borne out by Fig. 6, which differs from Fig. 5 mainly in the appearance of the new state at $\sigma_{T}=1$. Such a state agrees with the prediction $S 1^{\prime}$ but would be forbidden for the independent-bilayer states which surround it, since these must all have $\sigma_{T}=p / q$ with $p$ even. ${ }^{30}$ Moreover, it is further supported by the reappearance of the selection rule $\left|p q^{\prime}-p^{\prime} q\right|=1$ for the transitions $\frac{6}{5} \rightarrow 1$ and $1 \rightarrow \frac{4}{5}$ (or the $\frac{6}{7}$ state indicated in Fig. 6, though there is no well developed plateau for that state), as dictated by prediction $S 2^{\prime}$. A gold-plated signal would be the observation of the trajectory taken in the complex $\sigma_{T}$ plane during these transitions, since this should be described by the semicircle trajectories predicted by $S 3^{\prime}$ in samples exhibiting particle-hole symmetry.

For still smaller $d$ the behavior of the sample continues to evolve. This may be seen from the resistance traces given in 


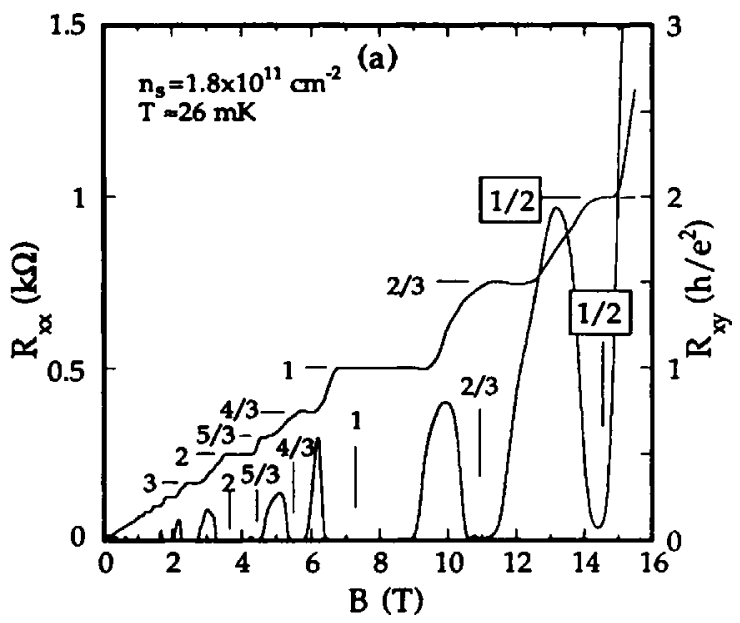

FIG. 7. Resistance traces for the same bilayer system as in Figs. 5 and 6 , but with still smaller $n_{s}$ and so smaller effective $d$. Data taken from Fig. 3 of Suen et al. (Ref. 23).

Fig. 7, which differ considerably from those of Figs. 5 and 6. This figure agrees in many ways with what would be expected from the trajectory of Fig. 3, including a phase of interlayer Coulomb correlations, although there are also a few troubling aspects to this description, as we now describe.

As discussed earlier, we qualitatively expect that shrinking $d$ causes $\Delta$ to grow, and this should increase the value of the field, $B_{c}$, at which the transition occurs from $\Delta$ domination to domination by $V_{c}^{11}$. We also expect the regime of independent bilayers to become narrower, and perhaps disappear, depending on how quickly $\Delta$ grows compared with $V_{c}^{12}$.

Whether these are borne out by Fig. 7 depends on the regime with which each of the plateaux is associated. Indeed all of the observed plateaux in Fig. 7, except for the one at $\sigma_{T}=\frac{1}{2}$, occur at fractions with odd denominators and so could be due to a tunneling-dominated monolayer $(M 1-M 3)$. On the other hand, both plateaux $\sigma_{T}=\frac{4}{3}$ and $\frac{2}{3}$ have even numerators and so could instead be interpreted as being due to the independent-bilayer category of phases $(S 1-S 3)$. These two options can be distinguished using the selection rule ( $M 2$ or $S 2$ ), and since Fig. 7 indicates this to be $\left|p q^{\prime}-p^{\prime} q\right|=1$-apart from the $\frac{5}{3} \rightarrow \frac{4}{3}$ transition, see below-it is the monolayer option which appears to be most appropriate. Under this interpretation there would be a direct transition between $\sigma_{T}$ $=\frac{2}{3}$ and $\sigma_{T}=\frac{1}{2}$ from the monolayer regime $(M 1-M 3)$ to the regime of interlayer Coulomb correlations $\left(S 1^{\prime}-S 3^{\prime}\right)$. If this picture is right then monolayer-type semicircle trajectories (M3) are predicted for all of the transitions in particle-hole symmetric samples, at least up to $\sigma_{T}=1$.

The $\frac{5}{3} \rightarrow \frac{4}{3}$ transition is the only exception to the selection rule $\left|p q^{\prime}-p^{\prime} q\right|=1$, and the interpretation of this transition is problematic within the present framework. However, this could indicate the existence of a poorly resolved state in between these two plateaux, similar to the way the incipient $\sigma_{T}=1$ state causes a funny selection rule between the $\frac{6}{5}$ and $\frac{6}{7}$ states in Fig. 5. A more baroque interpretation takes the $\frac{5}{3}$ $\rightarrow \frac{4}{3}$ transition to mark the point where the transition is made

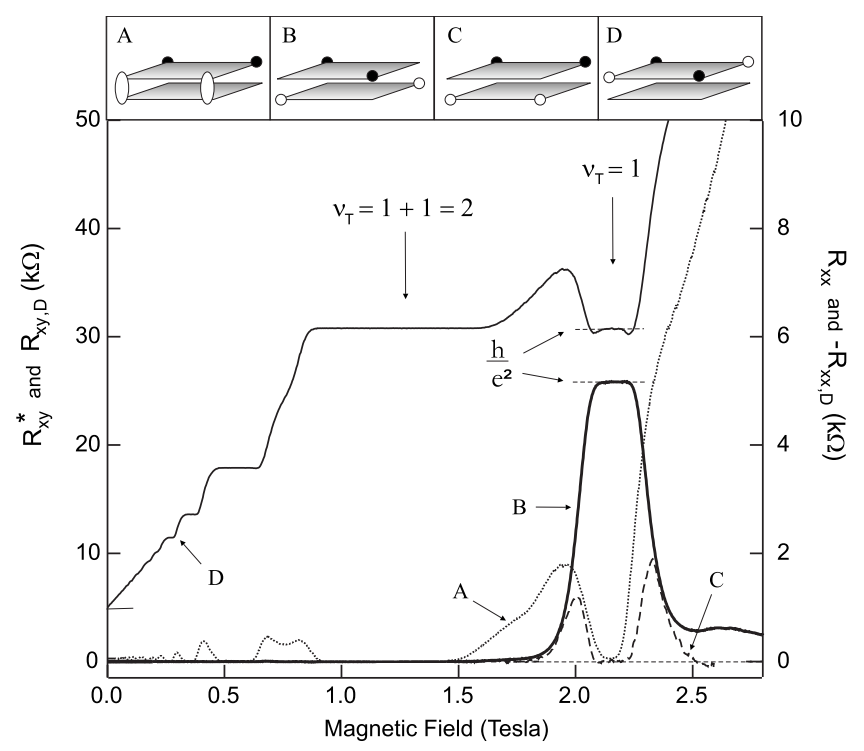

FIG. 8. Resistance traces for the bilayer systems reproduced from Ref. 25. The sign of trace $C$ is reversed in this plot.

into the independent-bilayer regime $(S 1-S 3)$, in which case Fig. 7 would require a further transition to the correlated bilayer regime $\left(S 1^{\prime}-S 3^{\prime}\right)$ for the $\sigma_{T}=1$ state, followed by a reentrant independent-bilayer state at $\sigma_{T}=\frac{2}{3}$. Such a picture would, however, also require a second transition to the correlated regime to account for the $\sigma_{T}=\frac{1}{2}$ state and we shall not pursue this possibility further here.

This analysis shows how the fact that there are three kinds of predictions may be used both to reconstruct the regime which is relevant, and to test the implications of the underlying $\Gamma_{0}(2)$ symmetry of each quantum Hall layer.

\section{B. Resolved bilayer systems}

The previous discussion involved only measurements of the total conductivity, $\sigma_{T}$, but more can be said if both $\sigma$ and $\widetilde{\sigma}$ are separately known. We now describe the implications of the symmetry for one such a measurement.

Reference 25 examines a crossover between two different plateaux - one with total filling factor $\nu_{T}=2$ and the other with $\nu_{T}=1$ - and explicitly give enough information to determine separately all of the complex components of the resistivity tensor, $\rho$ and $\tilde{\rho}$ as follows. Figure 8 reproduces the four resistance traces, $A, B, C$, and $D$, given in Ref. 25, where the sign of trace $C$ is reversed. Traces $B$ and $C$ directly give the interlayer resistivities, ${ }^{31}$

$$
\rho_{B}=\tilde{\rho}_{x y} \quad \text { and } \quad \rho_{C}=-\tilde{\rho}_{x x},
$$

while $A$ and $D$ represent intralayer resistivities,

$$
\rho_{D}=\rho_{x y} .
$$

The extraction of the intralayer Ohmic resistivity, $\rho_{x x}$, from the data is a little less direct. Denoting the $2 \times 2 \mathrm{com}$ plex resistivity tensor by 


$$
-\Sigma^{-1}=R=\left(\begin{array}{cc}
\rho & \tilde{\rho} \\
\tilde{\rho} & \rho
\end{array}\right)
$$

then the eigenvalues of $R$,

$$
\rho_{ \pm}=\rho \pm \tilde{\rho},
$$

are related to the corresponding eigenvalues of $\Sigma$ by

$$
\sigma_{ \pm}=\sigma \pm \widetilde{\sigma}=-\frac{1}{\rho_{ \pm}} .
$$

Trace $A$ of Fig. 8 then measures the Ohmic resistance corresponding to the total conductivity

$$
\sigma_{T}=2(\sigma+\widetilde{\sigma})=2 \sigma_{+}=-\frac{2}{\rho_{+}} .
$$

Consequently $\rho_{T}=-1 / \sigma_{T}=\rho_{+} / 2$, and so trace $A$ gives $\rho_{A}$ $=\rho_{+x x} / 2$, or

$$
\rho_{x x}=2 \rho_{A}+\rho_{C} .
$$

Given these resistivities, we see that the two plateaux observed in Ref. 25 occur for

$$
R_{1}=\left(\begin{array}{ll}
1 & 0 \\
0 & 1
\end{array}\right) \text { and } R_{2}=\left(\begin{array}{ll}
1 & 1 \\
1 & 1
\end{array}\right)
$$

which, respectively, correspond to $\left(\rho_{+}, \rho_{-}\right)_{1}=(1,1)$ and $\left(\rho_{+}, \rho_{-}\right)_{2}=(2,0)$, and so $\left(\sigma_{+}, \sigma_{-}\right)_{1}=(-1,-1)$ and $\left(\sigma_{+}, \sigma_{-}\right)_{2}=$ $\left(-\frac{1}{2}, \infty\right)$. Notice that $R_{2}$ is not invertible.

According to our proposal, the action of $\operatorname{Sp}(4, Z)$ on the bilayer system should manifest itself as $\Gamma_{0}(2)$ acting separately on $\sigma_{+}$and $\sigma_{-}$, with implications $B 1-B 3$ or $B 1^{\prime}-B 3^{\prime}$, depending on the category of flows between phases in which the system lies. For $\sigma_{ \pm}=p_{ \pm} / q_{ \pm}$prediction $B 1$ means, in particular, that $q_{ \pm}$is odd while $B 1^{\prime}$ implies $p_{ \pm}$is odd while $q_{ \pm}$is even. We see from this that $\sigma_{+}=\sigma_{-}=-1$ corresponds to condition $B 1$ while $\sigma_{+}=-\frac{1}{2}$ corresponds to $B 1^{\prime}$, and so the tran- sition observed in Ref. 25 is between the two different categories of bilayer flows.

Because the transition is not purely within one category or another we have no right to expect either of the sets of predictions $B 1-B 3$ or $B 1^{\prime}-B 3^{\prime}$ to apply. Interestingly enough prediction $B 2$ does still seem to apply, since both $\sigma_{+}:-1 \rightarrow$ $-\frac{1}{2}$ and $\sigma_{-}:-1 \rightarrow \infty$ satisfy the selection rule $\left|p q^{\prime}-p^{\prime} q\right|=1$ if we interpret $\infty=1 / 0$.

\section{CONCLUSIONS}

We have seen that the emergence of modular symmetries in two-dimensional semiconductors has striking observational consequences both for bilayer and spin-degenerate quantum Hall systems, just as it did in the better-established case of monolayer semiconducting heterostructures. These consequences include predictions for the pattern of allowed Hall plateaux; predictions for selection rules restricting which plateaux can be obtained from which by varying magnetic fields and charge densities at low temperatures; and, for samples with particle-hole symmetry, semicircle laws for the trajectories taken through the conductivity plane during these transitions. Experimental verification of these predictions would provide support for the picture that such modular symmetries are to be expected on very general grounds for two-dimensional systems. We hope these observations will stimulate experimental tests of these predictions.

\section{ACKNOWLEDGMENTS}

We thank M. Hilke for helpful discussions. Our research has been assisted by research funds provided by NSERC (Canada), the Killam Foundation, McGill and McMaster Universities, and Enterprise Ireland (E.I. Basic Research Grant No. SC/1998/739). B.P.D. wishes to thank the Perimeter Institute and C.P.B. thanks the Dublin Institute for Advanced Studies for their kind hospitality at various times while these ideas were being developed. *cburgess@perimeterinstitute.ca

†bdolan@thphys.nuim.ie

${ }^{1}$ D. C. Tsui, Physica B 164, 59 (1990).

${ }^{2}$ J. P. Eisenstein, M. P. Lilly, K. B. Cooper, L. N. Pfeiffer, and K. W. West, Physica E (Amsterdam) 6, 29 (2000).

${ }^{3}$ S. H. Simon, Solid State Commun. 134, 81 (2005).

${ }^{4}$ R. B. Laughlin, Phys. Rev. Lett. 50, 1395 (1983).

${ }^{5}$ J. Polchinski, Effective Field Theory of the Fermi Surface, presented at TASI 92, Boulder, CO, 3-28 June 1992; [Report No. TASI 92:0235-276 (QCD161:T45:1992)]; R. Shankar, Rev. Mod. Phys. 66, 129 (1994);

${ }^{6}$ A. Shapere and F. Wilczek, Nucl. Phys. B 320, 669 (1989).

${ }^{7}$ C. A. Lütken and G. G. Ross, Phys. Rev. B 45, 11837 (1992); 48, 2500 (1993).

${ }^{8}$ C. P. Burgess, Rim Dib, and B. P. Dolan, Phys. Rev. B 62, 15359 (2000); C. P. Burgess and C. A. Lutken, Phys. Lett. B 451, 365 (1999);
${ }^{9}$ D.-H. Lee, S. Kivelson, and S.-C. Zhang, Phys. Rev. Lett. 68, 2386 (1992); S. Kivelson, D.-H. Lee, and S.-C. Zhang, Phys. Rev. B 46, 2223 (1992).

${ }^{10}$ C. P. Burgess and B. P. Dolan, Phys. Rev. B 63, 155309 (2001).

${ }^{11}$ E. Witten, in From Fields to Strings, edited by M. Shifman, A. Vainshtein, and J. Wheater (World Scientific, Singapore, 2004), Vol. 2, p. 1173.

${ }^{12}$ C. P. Burgess and B. P. Dolan, Phys. Rev. B 65, 155323 (2002).

${ }^{13}$ D. Shahar, D. C. Tsui, and M. Shayegan, Science 274, 589 (1996).

${ }^{14}$ B. P. Dolan, Phys. Rev. B 62, 10278 (2000).

${ }^{15}$ J. K. Jain, Phys. Rev. Lett. 63, 199 (1989); J. K. Kain, S. A. Kivelson, and N. Trivedi, ibid. 64, 1297 (1990); J. K. Jain, Phys. Rev. B 41, 7653 (1990); J. K. Jain and V. J. Goldman, ibid. 45, 1255 (1992).

${ }^{16}$ C. P. Burgess and C. A. Lütken, Nucl. Phys. B 500, 367 (1997); B. P. Dolan, J. Phys. A 32, L243 (1999). 
${ }^{17}$ D. E. Khmel'nitskii, Pis'ma Zh. Eksp. Teor. Fiz. 38, 454 (1983). [JETP Lett. 38, 552 (1983)].

${ }^{18}$ A. M. M. Pruisken, Phys. Rev. Lett. 61, 1297 (1988).

${ }^{19}$ S. S. Murzin, M. Weiss, A. G. M. Jansen, and K. Eberl, Phys. Rev. B 66, 233314 (2002).

${ }^{20}$ A. H. MacDonald, P. M. Platzman, and G. S. Boebinger, Phys. Rev. Lett. 65, 775 (1990).

${ }^{21}$ C. P. Burgess and Brian P. Dolan, Phys. Rev. B 76, 113406 (2007).

${ }^{22}$ N. Koblitz, Introduction to Elliptic Curves and Modular Forms, 2nd ed., Graduate Texts in Mathematics Vol. 97 (SpringerVerlag, Berlin, 1984).

${ }^{23}$ Y. W. Suen, L. W. Engel, M. B. Santos, M. Shayegan, and D. C. Tsui, Phys. Rev. Lett. 68, 1379 (1992).

${ }^{24}$ J. P. Eisenstein, G. S. Boebinger, L. N. Pfeiffer, K. W. West, and Song He, Phys. Rev. Lett. 68, 1383 (1992).
${ }^{25}$ M. Kellogg, I. B. Spielman, J. P. Eisenstein, L. N. Pfeiffer, and K. W. West, Phys. Rev. Lett. 88, 126804 (2002).

${ }^{26}$ S. M. Girvin and A. H. MacDonald, Report No. IUCM95-012 (unpublished); arXiv:cond-mat/9505087.

${ }^{27}$ The notation is that of Ref. 22.

${ }^{28}$ Recall here that $\sigma_{+}=$odd/even so we may write $\sigma_{+}=k / 2 l$ where $k$ is odd. Then $\sigma_{T}=2 \sigma_{+}=k / l$, and so $k^{\prime}(2 l)-k\left(2 l^{\prime}\right)= \pm 2$ implies $k^{\prime} l-k l^{\prime}= \pm 1$.

${ }^{29}$ Alternatively, if $\Gamma_{\theta}$ is the relevant group — as appears to be the case for graphene-the analogs of $S 1-S 3$ are required for this group (Ref. 21).

${ }^{30}$ More evidence that the $\sigma_{T}=1$ plateau is due to interlayer correlations comes from its fragility to tilting the applied magnetic field (Ref. 26).

${ }^{31}$ The conversion from resistance to resistivity is immediate since the sample used in Ref. 25 is square. 